\title{
Usefulness of intraoperative determination of central lymph node metastasis by palpation in papillary thyroid cancer
}

\author{
Wan Wook Kim ${ }^{1}$, Jeeyeon Lee ${ }^{1}$, Jin Hyang Jung ${ }^{1}$, Ho Yong Park ${ }^{1}$, Won Hwa Kim ${ }^{2}$, Hye Jung Kim ${ }^{2}$, \\ Ji-Young Park ${ }^{3}$, Ralph P. Tufano ${ }^{4}$
}

${ }^{1}$ Department of Surgery, Kyungpook National University Chilgok Hospital, School of Medicine, Kyungpook National University, Daegu, Korea ${ }^{2}$ Department of Radiology, Kyungpook National University Chilgok Hospital, School of Medicine, Kyungpook National University, Daegu, Korea ${ }^{3}$ Department of Pathology, Kyungpook National University Chilgok Hospital, School of Medicine, Kyungpook National University, Daegu, Korea ${ }^{4}$ Department of Otolaryngology-Head and Neck Surgery, Johns Hopkins University School of Medicine, Baltimore, MD, USA

Received: March 4, 2020

Revised: April 3, 2020

Accepted: April 7, 2020

Corresponding author:

Wan Wook Kim

Department of Surgery, Kyungpook

National University Chilgok

Hospital, School of Medicine,

Kyungpook National University, 807

Hoguk-ro, Buk-gu, Daegu 41404,

Korea

Tel: +82-53-200-2705

Fax: +82-53-200-2027

E-mail: kww1324@naver.com
Background: This study evaluated the usefulness of judgment of central lymph node (LN) metastasis by surgeon's palpation in papillary thyroid cancer.

Methods: This study included 127 patients who underwent thyroidectomy and central compartment node dissection between October 2014 and February 2015. The criterion for suspicious LNs was hardness.

Results: Of the 20.5\% (28/127) of suspicious for metastatic LNs according to surgeon determination, $92.8 \%$ (26/28) were confirmed to be metastatic in the final pathological examinations. Metastatic LNs were found in $38(38.3 \%)$ of 99 patients without suspicious LNs, 29 of whom (76.3\%) had micrometastases. The sensitivity, specificity, and positive and negative predictive values for the determination of LN metastasis by a surgeon were $40.6 \%, 96.8 \%, 92.9 \%$, and $61.6 \%$, respectively.

Conclusion: Determination of central LN metastasis by a surgeon's palpation may be useful to evaluate LNs owing to the high specificity and positive predictive values, especially in macrometastasis or high-risk LN disease.

Keywords: Determination; Lymph node; Metastasis; Palpation; Surgeon

\section{Introduction}

The incidence of papillary thyroid carcinoma (PTC) has increased globally [1]. Although PTC has a much better prognosis than those of many other cancers, cervical lymph node (LN) metastasis occurs in $30 \%-80 \%$ cases and reportedly increases local recurrence rates $[2,3]$.

Central compartment node dissection (CCND) allows accu- rate LN staging, reduces local recurrence by clearing metastatic $\mathrm{LN}$, and has a good effect on survival [4]. However, the indication and optimal extent of LN dissection for PTC remain controversial (prophylactic vs. therapeutic, ipsilateral vs. bilateral) because the sensitivity of ultrasonography (USG) and computed tomography (CT) for central cervical LN metastasis in thyroid cancer is low, with poor diagnostic performance $[5,6]$. In addition, prophylactic CCND may increase morbidity including hypocalcemia and re-

Copyright (C) 2020 Yeungnam University College of Medicine

This is an Open Access article distributed under the terms of the Creative Commons Attribution Non-Commercial License (http://creativecommons.org/licenses/by-nc/4.0/) which permits unrestricted non-commercial use, distribution, and reproduction in any medium, provided the original work is properly cited. 
current laryngeal nerve (RLN) palsy; thus, the clinical benefit of survival gained from CCND is unclear [7]. However, CCND performed by experienced, high-volume surgeons ( $>25$ total thyroidectomies/year) have relatively lower postoperative morbidity rates within the acceptable range [8].

LN metastasis is one of the important criteria for determining the extent of surgery (lobectomy vs. total thyroidectomy, ipsilateral vs. bilateral CCND) in thyroid cancer. Total thyroidectomy is preferred to lobectomy if unilateral cancer has multiple central LN metastases, and bilateral CCND may be required rather than ipsilateral CCND. A study of central LN metastasis of unilateral cancer with clinically node-negative status in our institution reported that ipsilateral LN metastasis was the most important high-risk factor for predicting contralateral LN metastasis [9].

Intraoperative frozen biopsy is a useful method to determine the extent of surgery to minimize unnecessary bilateral CCND by confirming LN metastasis. Although frozen biopsy is available in tertiary hospitals with sufficient pathologist support, it is not a feasible option in most other hospitals. In addition, frozen biopsy leads to longer operation times and increases costs for examination; moreover, pathologist skills and efforts are important; thus, there are rarely false-negative results in frozen biopsy.

Therefore, our research team questioned whether inspection and palpation by the surgeon could confirm central LN metastasis and replace the need for frozen biopsy during surgery. Hence, this study aimed to evaluate the accuracy and usefulness of single-surgeon inspection and palpation to evaluate central LN metastasis and determine the extent of surgery.

\section{Materials and methods}

This study was approved by the Institutional Review Board (IRB) of the Kyungpook National University Chilgok Hospital (IRB No: 2020-04-008).

Since 2010, the author (WWK) has performed approximately 300-400 thyroid surgeries per year. To evaluate whether the metastasis can be judged by inspection and palpation, for approximately 2 months before starting the study, all LNs were examined using the same methods after CCND and frozen biopsy was performed to confirm the presence of metastasis in suspicious LNs. This study retrospectively included 127 consecutive patients who underwent thyroidectomy and CCND between October 2014 to February 2015. All patients were diagnosed with PTC based on fine-needle aspiration biopsy before surgery and clinically negative LN (nonspecific or considered to be benign) by physical examination and high-resolution USG. All USGs were performed by a well-trained thyroid surgeon or a radiologist specializing in thyroids. The exclusion criteria were benign or follicular neoplasm or other malignancy, previous thyroid operation, clinically positive LN (suspicious metastatic LN), or presence of central/ lateral LN metastasis. Regarding the extent of CCND, ipsilateral CCND was performed for unilateral PTC measuring less than 1 $\mathrm{cm}$ with negative LN, while bilateral CCND was performed in PTC with bilateral involvement or metastasis in ipsilateral central LN or grossly extrathyroidal extension. Ipsilateral CCND included pretracheal, paratracheal, and prelaryngeal LNs. Micrometastasis was defined as a maximum metastatic focus size $<0.2 \mathrm{~cm}$, while $\geq 0.2 \mathrm{~cm}$ was defined as macrometastasis. According to the American Thyroid Association Guidelines, all involved LNs sized $<0.2 \mathrm{~cm}$ and $<5$ metastatic LNs were classified as low-risk LN disease; whereas $>5$ metastatic LNs, macroscopic LN metastasis (clinical N1), and $\geq 3 \mathrm{~cm}$ metastatic LNs were considered highrisk LN disease. One specialized thyroid surgeon (WWK) examined each LN and assessed the metastasis after CCND by inspection and palpation. The surgeons divided the LNs into benign and suspicious metastatic groups and only the suspicious metastatic LNs were numbered. The results were confirmed by frozen or permanent biopsy. In the surgeon's experience, the critical criterion for suspicious metastatic LN was hardness ( $\geq 6$ points out of 10) rather than enlargement or discoloration. The sensitivity, specificity, positive predictive value (PPV), and negative predictive value (NPV) of intraoperative determinations by the surgeon were examined on basis of the final pathology results. All statistics were analyzed using IMB SPSS version 20.0 (IBM Corp., Armonk, NY, USA).

\section{Results}

The clinicopathologic results of the 127 patients are summarized in Table 1. A mean of 6.09 \pm 5.25 (range, 0-24) LNs was retrieved and a mean of $1.37 \pm 2.57$ (range, $0-17$ ) LNs were metastatic. LN metastases were present in 64 patients (50.4\%), of whom 37 (57.8\%) showed micrometastases, with a mean metastatic LN size of $0.28 \pm 0.24 \mathrm{~cm}$ (range, $0.1-1.2 \mathrm{~cm}$ ). The intraoperative determinations and pathologic results of all patients are shown (Fig. 1). Twenty-eight patients (22.0\%) had suspicious metastatic LNs based on the surgeon's determination, and a total of 128 suspicious metastatic LNs were harvested. A mean of $0.33 \pm 0.8$ (range, $0-5$ ) suspicious metastatic LNs were removed per patient. Of these 28 patients, 26 (92.9\%) were diagnosed with metastasis based on permanent pathologic examination (Fig. 1). Eighteen (69.2\%) and eight patients (30.8\%) had macrometastasis and micrometastasis, respectively. High-risk nodal disease was found in 20 (71.4\%) of these patients, while low-risk LN disease 
Table 1. Clinicopathologic results of 127 patients

\begin{tabular}{lc}
\hline Characteristic & Value \\
\hline Age (yr) & $46.8 \pm 9.7(25-71)$ \\
Sex (female:male) & $107: 20(84.3: 15.7)$ \\
Lobectomy:total thyroidectomy & $68: 59(53.5: 46.5)$ \\
Tumor size (cm) & $0.83 \pm 0.6(0.2-3.8)$ \\
PTMC & $93(73.2)$ \\
Extrathyroidal extension & $64(50.4)$ \\
Thyroiditis & $50(39.4)$ \\
Suspicious for metastatic LN determined by surgeon & $28(20.5)$ \\
Number removed from suspicious for metastatic LN & $0.33 \pm 0.8(0-5)$ \\
Frozen biopsy for suspicious LN & $17(13.4)$ \\
Number of retrieved LN & $6.09 \pm 5.2(0-24)$ \\
Number of metastatic LN & $1.37 \pm 2.5(0-17)$ \\
Metastatic LN size (cm) & $0.28 \pm 0.24(0.1-1.2)$ \\
Metastasis on LN & $64(50.4)$ \\
Micrometastasis on LN & $37 / 64(57.8)$ \\
\hline
\end{tabular}

Values are presented as mean \pm standard deviation (range) or number (\%). PTMC, papillary thyroid microcarcinoma; LN, lymph node.

was found in six patients (21.4\%). Among 99 patients without suspicious metastatic LNs, 38 (38.4\%) had metastasis, 29 (76.3\%) had micrometastasis. All of these 29 patients had fewer than five micrometastases. Low-risk LN disease was found in 90 of the 99 patients (90.9\%), including 61 patients without metastasis, while high-risk LN disease was observed in nine (9.1\%) patients.

The diagnostic accuracy of the determination of central LN metastasis by surgeon inspection and palpation is shown in Table 2, including a $40.6 \%$ sensitivity (26/64), $96.8 \%$ specificity (61/63), 92.9\% PPV (26/28), and 61.6\% NPV (61/99). A patient with thyroiditis is likely to have multiple reactive LNs. It is difficult to distinguish metastasis by palpation as thyroiditis is a complicated factor. Therefore, 77 patients without thyroiditis were re-examined (Table 3). Of these, 12 patients had suspicious LNs and all were diagnosed with metastasis upon final pathological examination. Of the 65 patients considered to have normal LNs, 23 (35.3\%) had metastasis, with micrometastasis present in $18 \mathrm{pa}-$ tients $(18 / 23,78.2 \%)$. Furthermore, the 23 patients with false-negative biopsy results mostly had low-risk LN disease. In patients without thyroiditis, the results of central $\mathrm{LN}$ metastasis determined by the surgeon included $34.2 \%$ sensitivity $(12 / 35)$, $100 \%$ specificity ( $42 / 42), 100 \%$ PPV (12/12), and $64.6 \%$ NPV $(42 / 65)$. The false-negative, false-positive, and accuracy rates were $65.7 \%, 0 \%$, and $70.1 \%$, respectively. No recurrence was observed during the mean 61.2-month follow-up period (range, 58.5-64.1 months).

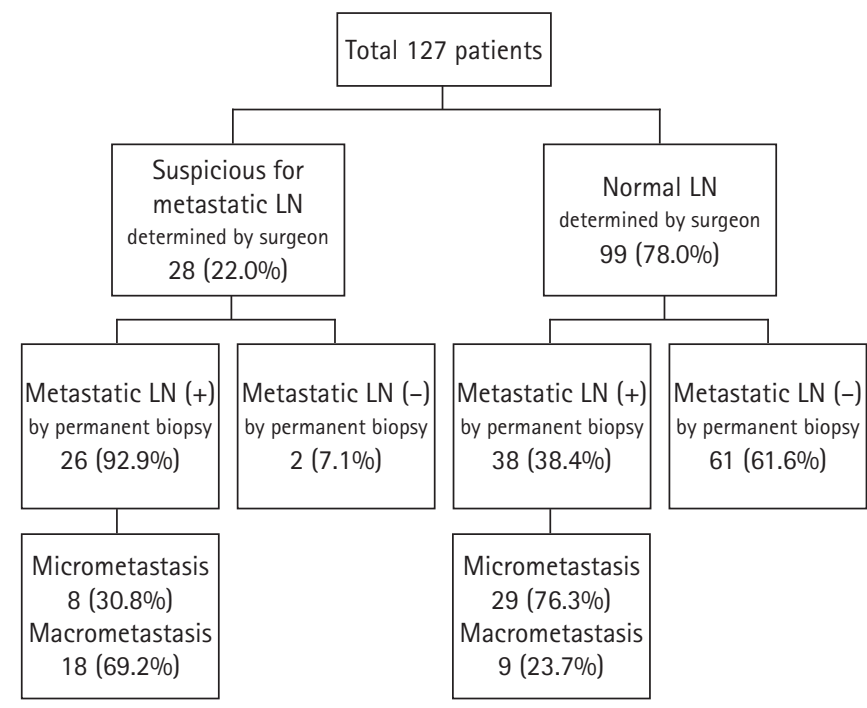

Fig. 1. Flowchart of lymph node (LN) assessment by palpation and pathology results of all 127 patients who underwent surgery.

\section{Discussion}

The extent of adequate CCND for differentiated thyroid carcinoma remains controversial. CCND reportedly improves patient survival rates and reduces the risk of recurrence when clinical LN metastasis is apparent $[10,11]$. However, concrete evidence is lacking to show that prophylactic CCND increases the survival rate in patients with differentiated thyroid cancer [12,13]. Although prophylactic CCND may be considered in patients aged $>45$ years and with T3/4 tumors, bilateral/multi-focal tumor, and extrathyroidal extension, and is not currently recommended for patients with clinical No, CCND has been reported to reduce postoperative recurrence rates and allow accurate nodal staging to establish postoperative management and follow-up strategies [14]. In addition, CCND has also been reported to lower thyroglobulin levels after surgery $[15,16]$.

LN metastasis in PTC, including micrometastasis, is commonly observed in approximately 30\%-80\% patients and is not uncommon in patients with clinical N0 on preoperative examination. LN metastasis is an important risk factor that should not be overlooked as it reportedly increases local recurrence rates and the risk of death in patients aged $\geq 45$ years [11]. The optimal treatment strategy is to accurately determine the central LN metastasis through preoperative examination, perform comprehensive CCND if LN metastasis is suspicious, decrease the recurrence rate, and improve the survival rate. In addition, it is essential to minimize complications such as hypocalcemia and RLN palsy by avoiding unnecessary bilateral CCND through appropriate CCND extent. 
Table 2. The diagnostic accuracy for determination of central lymph node (LN) metastasis by surgeon using inspection and palpation ( $\mathrm{n}=127)$

\begin{tabular}{lcc}
\hline Variable & \multicolumn{2}{c}{ Permanent pathology after surgery } \\
\cline { 2 - 3 } & Metastatic LN $(+)(n=64)$ & Metastatic LN (-) (n=63) \\
\hline Suspicious for metastatic LN determined by surgeon $(n=28)$ & $26(92.9)$ & $2(7.1)$ \\
Normal LN determined by surgeon $(n=99)$ & $38(38.4)$ & $61(61.6)$ \\
\hline
\end{tabular}

Values are presented as number (\%).

Table 3. The diagnostic accuracy for determination of central lymph node (LN) metastasis by surgeon in cases without thyroiditis $(n=77)$

\begin{tabular}{lcc}
\hline Variable & \multicolumn{2}{c}{ Permanent pathology after surgery } \\
\cline { 2 - 3 } & Metastatic LN $(+)(n=35)$ & Metastatic LN (-) ( $n=42)$ \\
\hline Suspicious for metastatic LN determined by surgeon $(n=12)$ & $12(100)$ & 0 \\
Normal LN determined by surgeon $(n=65)$ & $23(35.4)$ & $42(64.6)$ \\
\hline
\end{tabular}

Values are presented as number (\%).

However, surgeons find it challenging to decide whether to perform prophylactic CCND and determine the extent of CCND (ipsilateral/bilateral). LN evaluation using cervical USG is highly dependent on the examiner, as the diagnostic rate of USG for central cervical LN metastasis is as low as $20 \%-30 \%$ and those of other radiological examinations such as $\mathrm{CT}$, MRI, and positron emission tomography-CT are reported to be as low as $30 \%-40 \%$ $[17,18]$.

Frozen biopsy during thyroid surgery is useful to diagnose ambiguous thyroid cancer, confirm LN metastasis, and determine parathyroid gland histology. It also provides useful information to determine surgery extent. In our previous study, ipsilateral LN metastasis was the most potent high-risk factor for contralateral $\mathrm{LN}$ metastasis, suggesting that bilateral $\mathrm{LN}$ involvement is likely in these cases [7]. Therefore, total thyroidectomy and bilateral CCND may be good treatment options for multiple ipsilateral LN metastases on frozen section biopsy in patients with unilateral PTC to reduce the local recurrence rate by excising hidden metastatic LNs. However, frozen section biopsies require a clinically experienced pathologist and additional staff, along with increased cost and a minimum of 20-30 additional minutes for intraoperative examination. While waiting for frozen biopsy results, patients continue under extended anesthesia; prolonged operation time may have negative effects, especially in patients with comorbidities.

We have noted hardness or infiltration as a feature of metastatic LN from experience [19]. If the LN was not hard and enlarged, it was more likely to be reactive $\mathrm{LN}$ due to thyroiditis, as metastatic LNs are hard even if small. Black-colored LNs are not highly associated with metastasis. Because benign reactive LNs can also become large or discolored, enlargement or discoloration is not a direct feature of metastatic LNs. Although the hardness criterion was fairly subjective, we considered hardness greater than that of rubber (score $>6$ of 10 , where 10 is the hardest) to be suspicious. The more LNs surgeons palpate, the more clearly they could feel the difference. Benign LNs became softer when palpated while suspicious metastatic LNs remained firm even with continued palpation. As described in this study, surgeons can palpate and inspect all LNs after CCND and compare the results to the biopsy findings. The repetitive feedback will establish individual criteria based on the hardness for suspicious LNs.

In this study, it was difficult to distinguish between micrometastasis and benign LNs because the ratio of micrometastases was high and the metastatic LNs were small (mean size, $0.28 \pm 0.24$ $\mathrm{cm})$. Therefore, the sensitivity and NPV were low and surgeon inspection and palpation had limitations. However, metastatic LNs with macrometastasis or extranodal extension were easier to distinguish from benign LNs. Of the 38 patients with false-negative results (38.4\%), 29 (76.3\%) had micrometastasis and low-risk nodal disease. Therefore, the clinical significance of $\mathrm{LN}$ metastasis in false-negative cases may not be significant as it is likely to be low-risk LN disease. In cases with a strong belief of metastasis in the suspicious LN (large, hard, multiple LNs, or extranodal extension) by palpation, we recommend total thyroidectomy and both CCND.

Recently, extranodal extension has been reported as a high-risk factor associated with disease-free survival, cause-specific survival, recurrence, and metastasis $[20,21]$. It is the most important nodal risk factor as patients with extranodal extension are associated with disease progression showing high thyroglobulin, nodal persistence, and systemic disease. Two patients (both excluded from this study) who underwent modified radical neck dissection due to lateral LN metastasis during this period had LN metastasis with extranodal extension. All were suspected to be metastasis by the 
palpation method and all LNs biopsy results were confirmed to be metastases.

Approximately $40 \%$ of patients in this study had thyroiditis, with a large number of reactive LNs due to inflammatory reactions. Therefore, it is not easy to evaluate metastatic LNs by intraoperative palpation or preoperative USG in patients with thyroiditis. In this study, the sensitivity, specificity, PPV, and NPV of LN evaluation based on the surgeon's inspection and palpation were not reliable or accurate in patients with thyroiditis. However, in those without thyroiditis, the specificity and PPV of evaluation by LN palpation were $100 \%$. Therefore, LN evaluation using inspection and palpation may be useful in identifying metastasis in patients suspected of LN metastasis, especially in patients without thyroiditis.

Surgeon inspection and confirmation of each $\mathrm{LN}$ is helpful not only to assess whether metastatic $\mathrm{LN}$ is present but also to determine any unintentional removal of the parathyroid gland (incidental parathyroidectomy). It is important to reduce the incidence of permanent hypoparathyroidism by autotransplantation if unintended parathyroid removal is found during the inspection.

Our study has some limitations. First, there may be errors in the accuracy of the test because not all LNs were examined individually. Second, there is a limit to using judgment by palpation as an absolute criterion because it is difficult to establish objective criteria for hardness; thus, it requires subjective judgment. Third, LN evaluation by this method is difficult before CCND, thereby lowering the sensitivity. In this study, a single surgeon performed nodal palpation, and we did not assess interobserver variation in a blinded fashion. The determination of LNs by surgeon palpation may be helpful to some extent; however, it should be confined to an auxiliary diagnostic method due to some limitations.

The determination of central LN metastasis by surgeon's inspection and palpation could be used to evaluate LNs owing to its high specificity and PPV, especially for macrometastasis or highrisk $\mathrm{LN}$ disease.

\section{Acknowledgments}

\section{Conflicts of interest}

No potential conflict of interest relevant to this article was reported.

\section{Author contributions}

Conceptualization: HYP, WHK, HJK, JYP; Data curation: WWK, JHJ, JYP; Formal analysis: WWK, HYP; Methodology: JL; Investigation: WHK, HJK, JYP; Resources: HYP; Software: JYP; Supervision: HYP; Validation: WHK, HJK, JYP; Project ad- ministration: WHK; Visualization: RPT; Writing-original draft: WWK; Writing-review \& editing: WWK, JHJ, RPT.

\section{ORCID}

Wan Wook Kim, https://orcid.org/0000-0002-7363-5889

Jeeyeon Lee, https://orcid.org/0000-0003-1826-1690

Jin Hyang Jung, https://orcid.org/0000-0003-2607-1686

Ho Yong Park, https://orcid.org/0000-0002-4380-0089

Won Hwa Kim, https://orcid.org/0000-0001-7137-9968

Hye Jung Kim, https:/ / orcid.org/0000-0002-0263-0941

Ji-Young Park, https://orcid.org/0000-0002-7571-1064

Ralph P. Tufano, https://orcid.org/0000-0002-2219-3208

\section{References}

1. Ahn HS, Kim HJ, Welch HG. Korea's thyroid-cancer "epidemic": screening and overdiagnosis. N Engl J Med 2014;371: 1765-7.

2. Lim YC, Choi EC, Yoon YH, Kim EH, Koo BS. Central lymph node metastases in unilateral papillary thyroid microcarcinoma. Br J Surg 2009;96:253-7.

3. Ito Y, Kudo T, Kobayashi K, Miya A, Ichihara K, Miyauchi A. Prognostic factors for recurrence of papillary thyroid carcinoma in the lymph nodes, lung, and bone: analysis of 5,768 patients with average 10-year follow-up. World J Surg 2012;36:1274-8.

4. Adam MA, Pura J, Goffredo P, Dinan MA, Reed SD, Scheri RP, et al. Presence and number of lymph node metastases are associated with compromised survival for patients younger than age 45 years with papillary thyroid cancer. J Clin Oncol 2015;33: 2370-5.

5. Hwang HS, Orloff LA. Efficacy of preoperative neck ultrasound in the detection of cervical lymph node metastasis from thyroid cancer. Laryngoscope 2011;121:487-91.

6. Ahn JE, Lee JH, Yi JS, Shong YK, Hong SJ, Lee DH, et al. Diagnostic accuracy of CT and ultrasonography for evaluating metastatic cervical lymph nodes in patients with thyroid cancer. World J Surg 2008;32:1552-8.

7. Ito Y, Tomoda C, Uruno T, Takamura Y, Miya A, Kobayashi K, et al. Preoperative ultrasonographic examination for lymph node metastasis: usefulness when designing lymph node dissection for papillary microcarcinoma of the thyroid. World J Surg 2004;28:498-501.

8. Adam MA, Thomas S, Youngwirth L, Hyslop T, Reed SD, Scheri RP, et al. Is there a minimum number of thyroidectomies a surgeon should perform to optimize patient outcomes? Ann Surg 2017;265:402-7.

9. Kim WW, Park HY, Jung JH. Surgical extent of central lymph 
node dissection in clinically node-negative papillary thyroid cancer. Head Neck 2013;35:1616-20.

10. Zaydfudim V, Feurer ID, Griffin MR, Phay JE. The impact of lymph node involvement on survival in patients with papillary and follicular thyroid carcinoma. Surgery 2008;144:1070-7.

11. Yang L, Shen W, Sakamoto N. Population-based study evaluating and predicting the probability of death resulting from thyroid cancer and other causes among patients with thyroid cancer. J Clin Oncol 2013;31:468-74.

12. Barczyński M, Konturek A, Stopa M, Nowak W. Prophylactic central neck dissection for papillary thyroid cancer. Br J Surg 2013;100:410-8.

13. Moreno MA, Edeiken-Monroe BS, Siegel ER, Sherman SI, Clayman GL. In papillary thyroid cancer, preoperative central neck ultrasound detects only macroscopic surgical disease, but negative findings predict excellent long-term regional control and survival. Thyroid 2012;22:347-55.

14. Sancho JJ, Lennard TW, Paunovic I, Triponez F, Sitges-Serra A. Prophylactic central neck disection in papillary thyroid cancer: a consensus report of the European Society of Endocrine Surgeons (ESES). Langenbecks Arch Surg 2014;399:155- 63.

15. Wang TS, Evans DB, Fareau GG, Carroll T, Yen TW. Effect of prophylactic central compartment neck dissection on serum thyroglobulin and recommendations for adjuvant radioactive iodine in patients with differentiated thyroid cancer. Ann Surg
Oncol 2012;19:4217-22.

16. Hartl DM, Leboulleux S, Al Ghuzlan A, Baudin E, Chami L, Schlumberger M, et al. Optimization of staging of the neck with prophylactic central and lateral neck dissection for papillary thyroid carcinoma. Ann Surg 2012;255:777-83.

17. Jeong HS, Baek CH, Son YI, Choi JY, Kim HJ, Ko YH, et al. Integrated 18F-FDG PET/CT for the initial evaluation of cervical node level of patients with papillary thyroid carcinoma: comparison with ultrasound and contrast-enhanced CT. Clin Endocrinol (Oxf) 2006;65:402-7.

18. Weber AL, Randolph G, Aksoy FG. The thyroid and parathyroid glands. CT and MR imaging and correlation with pathology and clinical findings. Radiol Clin North Am 2000;38:110529.

19. Finn S, Toner M, Timon C. The node-negative neck: accuracy of clinical intraoperative lymph node assessment for metastatic disease in head and neck cancer. Laryngoscope 2002;112:630 3.

20. Lango M, Flieder D, Arrangoiz R, Veloski C, Yu JQ Li T, et al. Extranodal extension of metastatic papillary thyroid carcinoma: correlation with biochemical endpoints, nodal persistence, and systemic disease progression. Thyroid 2013;23:1099-105.

21. Wu MH, Shen WT, Gosnell J, Duh QY. Prognostic significance of extranodal extension of regional lymph node metastasis in papillary thyroid cancer. Head Neck 2015;37:1336-43. 